\title{
A wearable artificial kidney for patients with end-stage renal disease
}

\author{
Victor Gura, ${ }^{1,2}$ Matthew B. Rivara, ${ }^{3}$ Scott Bieber, ${ }^{3}$ Raj Munshi, ${ }^{3,4}$ Nancy Colobong Smith, ${ }^{3,5}$ \\ Lori Linke, ${ }^{3}$ John Kundzins, ${ }^{3}$ Masoud Beizai, ${ }^{6}$ Carlos Ezon, ${ }^{6}$ Larry Kessler, ${ }^{3,7}$ \\ and Jonathan Himmelfarb ${ }^{3}$ \\ ${ }^{1}$ Cedars-Sinai Medical Center, Los Angeles, California, USA. 'David Geffen School of Medicine at UCLA, UCLA, Los Angeles, \\ California, USA. ${ }^{3}$ Kidney Research Institute, Division of Nephrology, University of Washington, Seattle, Washington, USA. \\ ${ }^{4}$ Division of Nephrology, Department of Pediatrics, Seattle Children's Hospital and University of Washington, Seattle, \\ Washington, USA. ${ }^{5}$ University of Washington Medical Center, Seattle, Washington, USA. ${ }^{6}$ Blood Purification Technologies \\ Inc., Beverly Hills, California, USA. D. \\ Seattle, Washington, USA.
}

BACKGROUND. Stationary hemodialysis machines hinder mobility and limit activities of daily life during dialysis treatments. New hemodialysis technologies are needed to improve patient autonomy and enhance quality of life.

METHODS. We conducted a FDA-approved human trial of a wearable artificial kidney, a miniaturized, wearable hemodialysis machine, based on dialysate-regenerating sorbent technology. We aimed to determine the efficacy of the wearable artificial kidney in achieving solute, electrolyte, and volume homeostasis in up to 10 subjects over 24 hours.

RESULTS. During the study, all subjects remained hemodynamically stable, and there were no serious adverse events. Serum electrolytes and hemoglobin remained stable over the treatment period for all subjects. Fluid removal was consistent with prescribed ultrafiltration rates. Mean blood flow was $42 \pm 24 \mathrm{ml} / \mathrm{min}$, and mean dialysate flow was $43 \pm 20 \mathrm{ml} / \mathrm{min}$. Mean urea, creatinine, and phosphorus clearances over 24 hours were $17 \pm 10,16 \pm 8$, and $15 \pm 9 \mathrm{ml} / \mathrm{min}$, respectively. Mean $\beta_{2}$-microglobulin clearance was $5 \pm 4 \mathrm{ml} / \mathrm{min}$. Of 7 enrolled subjects, 5 completed the planned 24 hours of study treatment. The trial was stopped after the seventh subject due to device-related technical problems, including excessive carbon dioxide bubbles in the dialysate circuit and variable blood and dialysate flows.

Authorship note: V. Gura and M.B. Rivara contributed equally to this work.

Conflict of interest: $V$. Gura is Chief Medical Officer and a stockholder of Blood Purification Technologies Inc. M. Beizai and C. Ezon are employees of Blood Purification Technologies Inc M. Beizai and C. Ezon are listed as inventors on a patent related to the research described in this manuscript (patent US8034161). V. Gura is listed as an inventor on patents related to the research described in this manuscript (patent US8034161; US6960179; US7597677; US7645253; US7309323; US7854718).

Submitted: February 23, 2016

Accepted: May 4, 2016

Published: June 2, 2016

Reference information:

CONCLUSION. Treatment with the wearable artificial kidney was well tolerated and resulted in effective uremic solute clearance and maintenance of electrolyte and fluid homeostasis. These results serve as proof of concept that, after redesign to overcome observed technical problems, a wearable artificial kidney can be developed as a viable novel alternative dialysis technology.

TRIAL REGISTRATION. ClinicalTrials.gov NCT02280005.

FUNDING. The Wearable Artificial Kidney Foundation and Blood Purification Technologies Inc.

\section{Introduction}

Worldwide, the prevalence of end-stage renal disease (ESRD) treated with maintenance dialysis exceeds 2 million individuals (1). These patients suffer from an exceptionally high burden of morbidity and mortality. Adjusted rates of all-cause mortality are up to 8 times greater for dialysis patients compared with age-matched individuals in the general population (2-4). Current hemodialysis therapies require patients to adhere to restrictive dietary and fluid intake limitations and are associated with a high pill burden (57). Recognition of these limitations has prompted a search for alternatives to conventional thrice-weekly hemodialysis that may improve patient-centered clinical outcomes, including survival, treatment burden, and quality of life. Accumulating evidence suggests that longer and/or more frequent dialysis may benefit patients through improvements in metabolic parameters, reduced left ventricular mass, and greater blood pressure control (8-14). However, current hemodialysis machines are stationary and as such substantially 
limit freedom of movement of patients and their ability to engage in activities of daily living. Furthermore, functional capability after dialysis is frequently hindered by severe fatigue $(15,16)$. As a consequence, few patients undergoing maintenance dialysis are fully rehabilitated, leading to a high prevalence of poor health-related quality of life within this vulnerable population (17-19).

There is thus a critical unmet need for new dialysis technologies that offer patients an enhanced spectrum of choices and may address some of the key limitations of the current ESRD treatment paradigm. An alternative with the potential to improve on current hemodialysis technologies is a continuously operating and wearable artificial kidney (WAK) designed to provide continuous solute clearance and ultrafiltration capacity (20). We have previously described the development of such a device, which utilizes dialysate-regenerating sorbent technology paired with a miniaturized, dual-channel, battery-operated, pulsatile pump for driving both blood and dialysate simultaneously (20). This pulsatile pump generates a unique flow pattern that enhances convective transfer of solutes across the dialyzer membrane. Previous pilot studies of the WAK have demonstrated its short-term safety and efficacy in solute clearance and fluid removal but have been limited to treatment durations of less than 8 hours (21-23).

Here, we report the results of a 24-hour exposure of the WAK in humans. The primary goals of this study were to test the safety and efficacy of the WAK in achieving solute clearance, electrolyte homeostasis, and volume removal over a continuous 24 -hour period. Additionally, we sought to evaluate treatment-related satisfaction and quality of life with the WAK and compare these ratings to those for conventional in-center hemodialysis treatments among patients exposed to the device.

\section{Results}

This was a prospective, nonrandomized, exploratory clinical trial (NCT02280005), in which up to 10 subjects could receive therapy with the WAK for 24 hours while being monitored in a hospital setting. Development of the WAK was supported via collaboration with the US FDA Center for Devices and Radiological Health through its Innovation Pathway 2.0 (24).

Subject characteristics. Figure 1 depicts the flow of participants in the trial. Of 411 patients initially screened, 152 met study eligibility criteria (37\%); of these, 24 were approached, and $11(46 \%)$ consented to study participation. Table 1 shows the baseline demographic and laboratory data for all study subjects at the time of enrollment. Overall, the mean age was 49 years (range 27-73 years), 4 of the subjects were women, 3 had diabetes, and 3 had comorbid congestive heart failure. The average duration of maintenance hemodialysis prior to study enrollment was 15 months (range 3-41 months). Mean body mass index was 24 (range 17-31).

Solute clearance, electrolyte, and volume homeostasis. Mean concentrations of serum electrolytes over the 24-hour WAK treatment period remained stable (Table 2). Before starting treatment, mean serum potassium concentration was $3.8 \pm 0.5 \mathrm{meq} / 1$ and serum phosphorus concentration was $3.1 \pm 0.5 \mathrm{mg} / \mathrm{dl}$. At the end of the 24-hour WAK treatment, these concentrations were $3.9 \pm 0.4 \mathrm{meq} / 1$ and $3.9 \pm 0.8 \mathrm{mg} / \mathrm{dl}$, respectively $(P$ $=0.01$ for trend in serum phosphorus). During the study period, mean blood flow was $42 \pm 24 \mathrm{ml} / \mathrm{min}$ and dialysate flow was $43 \pm 20 \mathrm{ml} / \mathrm{min}$, with a moderate decrease in blood flows at between 16 and 24 hours. Mean weighted-average concentrations of blood urea nitrogen (BUN) were significantly lower during the 24-hour WAK treatment relative to weighted-average concentrations from a 48-hour period before the WAK encompassing conventional hemodialysis treatments $(17 \pm 5 \mathrm{mg} / \mathrm{dl}$ versus $39 \pm 18 \mathrm{mg} / \mathrm{dl}, P=0.002$ ) (Figure 2). Similarly, mean $\beta_{2}$-microglobulin concentrations were significantly lower during WAK treatment relative to periods of conventional hemodialysis therapy $(17 \pm 8 \mathrm{mg} / 1$ versus $22 \pm 11 \mathrm{mg} / 1, P=0.04)$.

Mean BUN, creatinine, and phosphorus clearances during the study period were $17 \pm 10,16 \pm 8$, and $15 \pm 9 \mathrm{ml} / \mathrm{min}$, respectively; mean $\beta_{2}$-microglobulin clearance was $5 \pm 4 \mathrm{ml} / \mathrm{min}$ (Figure 3 ). There was close concordance of blood-based and dialysate-based measurements of solute clearance (Figure 3 and Supplemental Table 1; supplemental material available online with this article; doi:10.1172/jci. insight.86397DS1), and there was a general moderate downward trend in clearance of all solutes over the 24-hour study period that closely paralleled a downward trend in blood flow measurements (Figures 4 and 5). Clearance of $\beta 2$-microglobulin was consistently lower than clearance of small solutes, irrespective of study time point.

Of the 5 subjects who completed the planned 24-hour treatment period, mean 24-hour ultrafiltration volume was $1,002 \pm 380 \mathrm{ml}$. Ultrafiltration was accomplished as intended, with a mean difference between prescribed and achieved ultrafiltration over the study period of $3 \pm 15 \mathrm{ml}$. Mean systolic blood pressure 


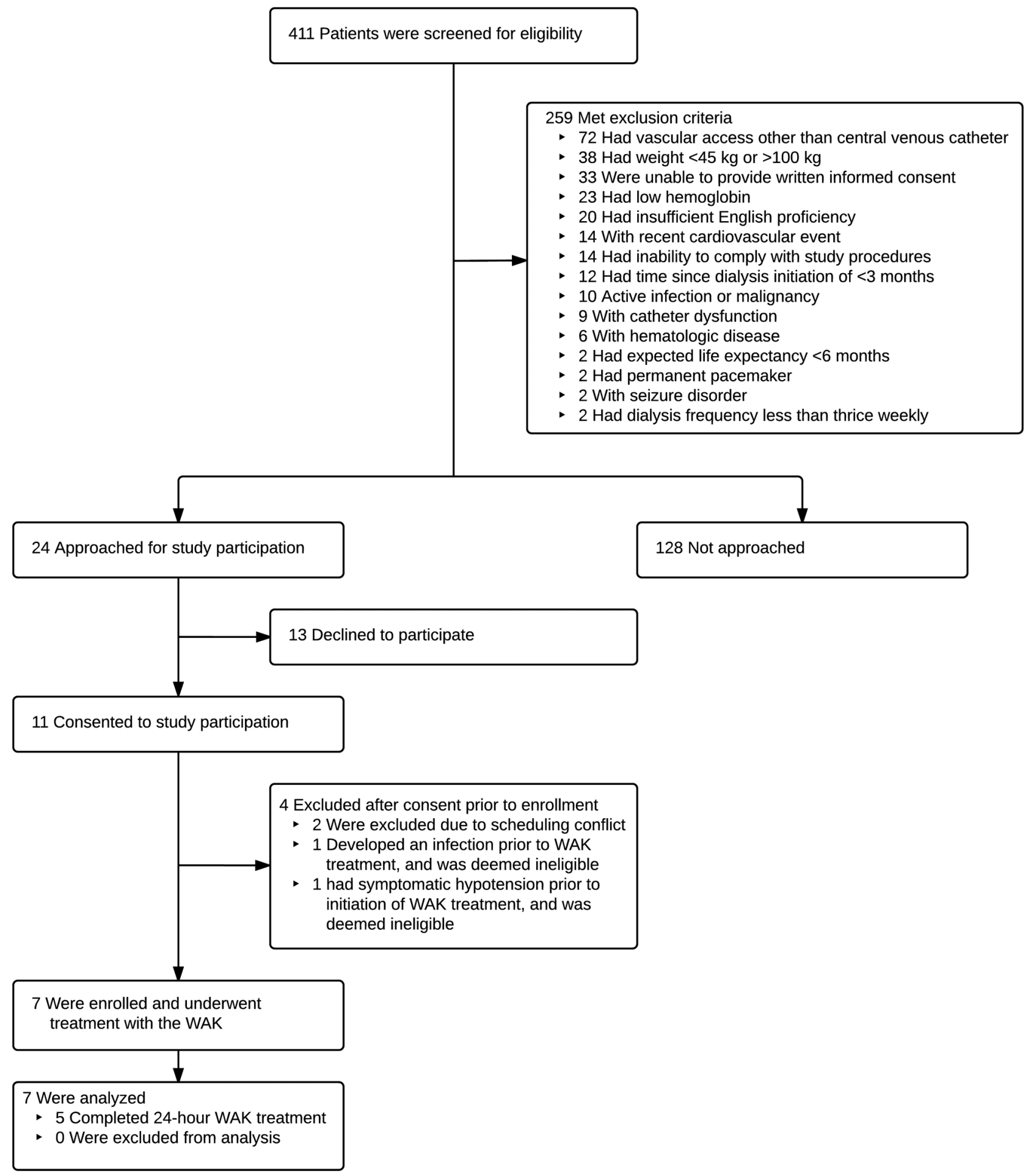

Figure 1. Study enrollment. WAK, wearable artificial kidney.

declined from $140 \pm 23 \mathrm{mmHg}$ at baseline to $127 \pm 6 \mathrm{mmHg}$ at the end of treatment, though this change was not statistically significant (Table 2). No subject was observed to have a systolic blood pressure of less than $100 \mathrm{mmHg}$ during the WAK treatment period, and only 1 subject was observed to have a systolic blood pressure of less than $120 \mathrm{mmHg}$.

Device performance and technical complications. Over the course of the 24-hour study period, dialysis-regenerating sorbents performed as expected, with no evidence of saturation with uremic solute (Supplemen- 
Table 1. Characteristics of the subjects at enrollment

\begin{tabular}{|c|c|}
\hline \multicolumn{2}{|l|}{ Characteristic } \\
\hline No. of subjects & 7 \\
\hline Age (range [yr]) & $49 \pm 14(27-73)$ \\
\hline \multicolumn{2}{|l|}{$\operatorname{Sex}(n[\%])$} \\
\hline Male & $3(43)$ \\
\hline Female & $4(57)$ \\
\hline \multicolumn{2}{|l|}{ Race $(n[\%])$} \\
\hline Black & $3(43)$ \\
\hline White & $3(43)$ \\
\hline Other & $1(14)$ \\
\hline \multirow[t]{2}{*}{ Body mass index (range) ${ }^{A}$} & $24 \pm 5$ \\
\hline & $(17-31)$ \\
\hline \multirow[t]{2}{*}{ Months since initiation of dialysis (range) } & $15 \pm 13$ \\
\hline & $(3-41)$ \\
\hline Diabetes ( $n[\%]$ ) & $3(43)$ \\
\hline Congestive heart failure ( $n[\%]$ ) & $3(43)$ \\
\hline Peripheral vascular disease ( $n[\%]$ ) & $3(43)$ \\
\hline \multicolumn{2}{|c|}{ Screening predialysis laboratory values (range) } \\
\hline Serum sodium (meq/l) & $140 \pm 1(137-141)$ \\
\hline Serum potassium (meq/l) & $4.9 \pm 0.8(3.7-5.8)$ \\
\hline Serum bicarbonate (meq/l) & $26 \pm 4(21-32)$ \\
\hline Serum albumin (g/dl) & $4.1 \pm 0.5(3.4-4.6)$ \\
\hline Serum blood urea nitrogen $(\mathrm{mg} / \mathrm{dl})$ & $62 \pm 19(41-90)$ \\
\hline Serum creatinine (mg/dl) & $8.5 \pm 1.8(6.3-11.9)$ \\
\hline Blood hemoglobin (g/dl) & $10.8 \pm 0.9(9.8-12.4)$ \\
\hline
\end{tabular}

Continuous variables are presented as mean $\pm \mathrm{SD}$. Categorical variables are presented as number (\%). ${ }^{\mathrm{A}}$ The body mass index is predialysis weight in kilograms divided by the square of the height in meters.

tal Table 2). In 6 of the 7 subjects, no significant clotting was observed, and mean 24-hour unfractionated heparin administration among all subjects was $956 \pm 218$ units. In 1 subject, treatment was discontinued after 4 hours due to clotting of the blood circuit following ambulation. In a second subject, WAK treatment was discontinued after 10 hours due to observed pink discoloration of the dialysate. This occurred after dialysate circuit reconnection for standard saline rinse, and there was no laboratory evidence of hemolysis. Elevated ammonia levels at hour 11 were detected in the dialysis circuit after passing through the sorbent cartridge for 1 subject, requiring replacement of the sorbent cartridges. No evidence of sorbent column ammonia saturation was detected in the remaining subjects. For 2 subjects, replacement of the main lithium ion battery was required prior to completion of the 24-hour treatment period. Removal of gas bubbles from the blood circuit was required in 3 study subjects. For these subjects, activation of the bubble detector on the venous limb of the blood circuit occurred early in the course of the WAK treatment period due to small bubbles that had likely remained in the dialyzer header following circuit priming. In each case, activation of the bubble detector sounded an audible alarm and automatically stopped the main shuttle pump. Study investigators then used a 3.0-ml lure-lock syringe to remove the bubbles using a venous limb sampling port prior to restarting the main pump. The trial was stopped after the seventh subject due to the cumulative occurrence of device-related technical problems, which included excessive carbon dioxide bubbles in the dialysate circuit, which exceeded degassing capacity, tubing kinks, and variable pump function, resulting in fluctuating blood and dialysate flow rates. A complete list of technical issues encountered and correction actions taken is detailed in Supplemental Table 3.

Adverse events and tolerability. There were no serious adverse events. Two subjects experienced mild muscle cramping, which in one case resolved spontaneously and in the other case resolved with a reduction in the delivered ultrafiltration rate (Supplemental Table 4). One subject experienced a brief episode of nausea, and one had an episode of diarrhea on the day following WAK treatment. One subject experienced moderate malaise after discontinuation of WAK treatment. This subject, who had an elevated white blood cell 
Table 2. Serum electrolytes, hematologic indices, and hemodynamic parameters over 24-hour study period ${ }^{\mathrm{A}}$

\begin{tabular}{|c|c|c|c|c|c|c|c|}
\hline \multirow[b]{2}{*}{ Serum electrolytes } & \multicolumn{7}{|c|}{ Time (h) } \\
\hline & $\begin{array}{l}\text { Baseline after } \\
\text { dialysis }\end{array}$ & 1 & 8 & 12 & 16 & 24 & $\begin{array}{l}P \text { value for } \\
\text { trend }\end{array}$ \\
\hline Sodium (meq/l) & $131 \pm 2$ & $131 \pm 2$ & $129 \pm 3$ & $130 \pm 4$ & $131 \pm 4$ & $135 \pm 4$ & 0.13 \\
\hline Potassium (meq/l) & $3.8 \pm 0.5$ & $3.7 \pm 0.5$ & $3.8 \pm 0.3$ & $3.8 \pm 0.4$ & $3.7 \pm 0.4$ & $3.9 \pm 0.4$ & 0.33 \\
\hline Chloride (meq/l) & $95 \pm 2$ & $96 \pm 2$ & $97 \pm 3$ & $96 \pm 3$ & $97 \pm 3$ & $95 \pm 4$ & 0.89 \\
\hline Total calcium (mg/dl) & $8.5 \pm 0.4$ & $8.7 \pm 0.6$ & $8.6 \pm 1.0$ & $8.5 \pm 0.8$ & $8.5 \pm 0.6$ & $8.6 \pm 0.7$ & 0.94 \\
\hline Phosphorus (mg/dl) & $3.1 \pm 0.5$ & $3.1 \pm 0.6$ & $3.6 \pm 0.9$ & $4.2 \pm 1.2$ & $4.2 \pm 1.2$ & $3.9 \pm 0.8$ & 0.01 \\
\hline \multicolumn{8}{|l|}{ Hematologic indices } \\
\hline Hemoglobin (g/dl) & $10.4 \pm 1.1$ & $11.2 \pm 1.4$ & $10.8 \pm 0.9$ & $10.7 \pm 1.1$ & $10.5 \pm 1.1$ & $11.1 \pm 1.1$ & 0.81 \\
\hline Lactate dehydrogenase (units/I) & $165 \pm 33$ & $183 \pm 49$ & $179 \pm 42$ & $177 \pm 40$ & $162 \pm 34$ & $191 \pm 35$ & 0.80 \\
\hline Haptoglobin (mg/dl) & $136 \pm 94$ & $175 \pm 95$ & - & $110 \pm 92$ & - & $93 \pm 96$ & 0.13 \\
\hline Diastolic blood pressure (mmHg) & $77 \pm 15$ & $82 \pm 19$ & $77 \pm 19$ & $75 \pm 21$ & $71 \pm 20$ & $78 \pm 18$ & 0.51 \\
\hline Heart rate (bpm) & $80 \pm 6$ & $79 \pm 7$ & $79 \pm 10$ & $77 \pm 10$ & $82 \pm 12$ & $88 \pm 10$ & 0.24 \\
\hline
\end{tabular}

count prior to WAK treatment, received a single prophylactic administration of intravenous antibiotics. No further antibiotics were administered, as blood cultures were negative for growth of bacterial or fungal organisms. Five subjects were noted to have premature ventricular contractions while monitored on continuous telemetry; there were no episodes of sustained atrial or ventricular arrhythmia. All 7 subjects were able to ambulate while receiving WAK treatment.

Treatment satisfaction and health-related quality of life. Comparing treatment satisfaction with conventional hemodialysis therapy versus satisfaction with their experience using the WAK, subjects reported significantly greater overall satisfaction with the WAK $(P<0.001)$. Individual Renal Treatment Satisfaction Questionnaire (RTSQ) item satisfaction scores were significantly higher for the WAK in 10 of 13 domains assessed, including satisfaction with treatment-related side effects, convenience and flexibility of treatment, discomfort or pain involved with treatment, and satisfaction with freedom afforded by the treatment modality (Table 3).

\section{Discussion}

Under the auspices of the FDA Innovation Pathway 2.0, we completed an exploratory clinical trial of the WAK - a miniaturized, wearable hemodialysis system providing continuous solute clearance and volume removal capacity for patients with ESRD. During the study, hemodynamic parameters remained stable, ultrafiltration was achieved as intended, and there were no unexpected adverse treatment effects. Additionally, solute clearances were maintained over 24 hours and closely mirrored achieved blood flow. Acid-base and electrolyte homeostasis were maintained without restriction on patients' dietary choices and without use of phosphorus-binding medications. Importantly, study subjects reported greater treatment satisfaction among multiple treatment-related domains with WAK treatment compared with conventional hemodialysis treatment. Furthermore, the ability to ambulate while undergoing dialysis, if further proven in additional studies, would liberate patients from the need to be tethered to a stationary machine during dialysis treatments. A variety of technical complications were observed in our study that will need to be addressed through device redesign and refinement to enhance device safety and reliability prior to further longer-term studies of the WAK in humans. However, our findings provide proof of concept that the WAK could be developed as a viable novel dialysis technology.

Efforts to develop a portable and wearable dialysis system date to the early 1970s, when Kolff and colleagues developed a wearable hemodialysis module that could be disconnected from an accompanying 



Figure 2. Mean plasma concentrations of blood urea nitrogen and $\beta_{2}$-microglobulin during conventional hemodialysis before WAK and paired WAK treatment study periods. Data are presented as mean \pm SD for each time point. (A) Blood urea nitrogen ( $\mathrm{mg} / \mathrm{dl})$. (B) Plasma $\beta_{2}$-microglobulin (mg/l). HD, hemodialysis; WAK, wearable artificial kidney.

20-liter dialysate bath for up to one-third of the cumulative dialysis time (25). This innovative device utilized a single needle, a dual-chamber pulsatile pump, and rechargeable nickel-cadmium batteries and allowed clinician adjustments to blood flow and ultrafiltration rates. However, Kolff's device suffered from the absence of a full sorbent system, relying instead on a charcoal canister only for adsorption of filtered toxins, and additionally required patients to be tethered to a stationary dialysate bath for at least two-thirds of any dialysis session. Additional early efforts to design and build a wearable renal replacement system explored the possibility of utilizing hemofiltration technology alone, without counter-current dialysate circulation, but were complicated in some cases by the need for patients to consume a large volume of replacement fluid, limiting practical application (26). The application of sorbent technology to a wearable hemodialysis system was initially proposed by Blumenkrantz and colleagues in the late 1970s as an extension of newly developed and commercially available dialysate-regenerating sorbent systems (27). Subsequently, Murisasco and colleagues developed a wearable hemofiltration device utilizing sorbent-containing minicartridges to minimize the requirement for excessive intake of replacement fluid; but this system required replacement of the minicartridges up to 4 times daily and further required patients to use transcutaneous arteriovenous shunts with conse-

quent risk for infection (28). These early technological innovations paved the way for the development of the WAK, which extends earlier efforts through incorporation of modern-day miniaturization technology, biocompatible dialyzer materials, battery design, and safety features. In addition to the WAK, the past decade has also seen efforts to design a number of other prototype next-generation wearable renal replacement therapies, which include wearable peritoneal dialysis systems and implantable renal assist devices (29-31), though none have yet undergone testing in clinical trials involving human subjects.

The WAK is designed to be worn and used by patients for up to 24 hours per day and, thus, represents a wearable form of extended-hours hemodialysis in which patients receive substantially longer treatments than available with conventional thrice-weekly hemodialysis. Extended-hours therapies have been shown to lead to improved control of electrolyte homeostasis, including reductions in serum phosphorus (32-34). Extended-hours therapies also allow for slower and more physiological rates of volume removal via ultrafiltration, which may reduce the frequency of intradialytic hypotensive events and intradialytic myocardial stunning (35-37). Additionally, longer weekly treatment times have been shown to result in increased dialytic clearance of larger-molecular weight and protein-bound uremic retention solutes, which may at least partly underlie the improved outcomes observed among patients undergoing extended-hours regimens (38, 39).

In our study, blood and dialysate flow, and consequently solute clearance, were intentionally lower 

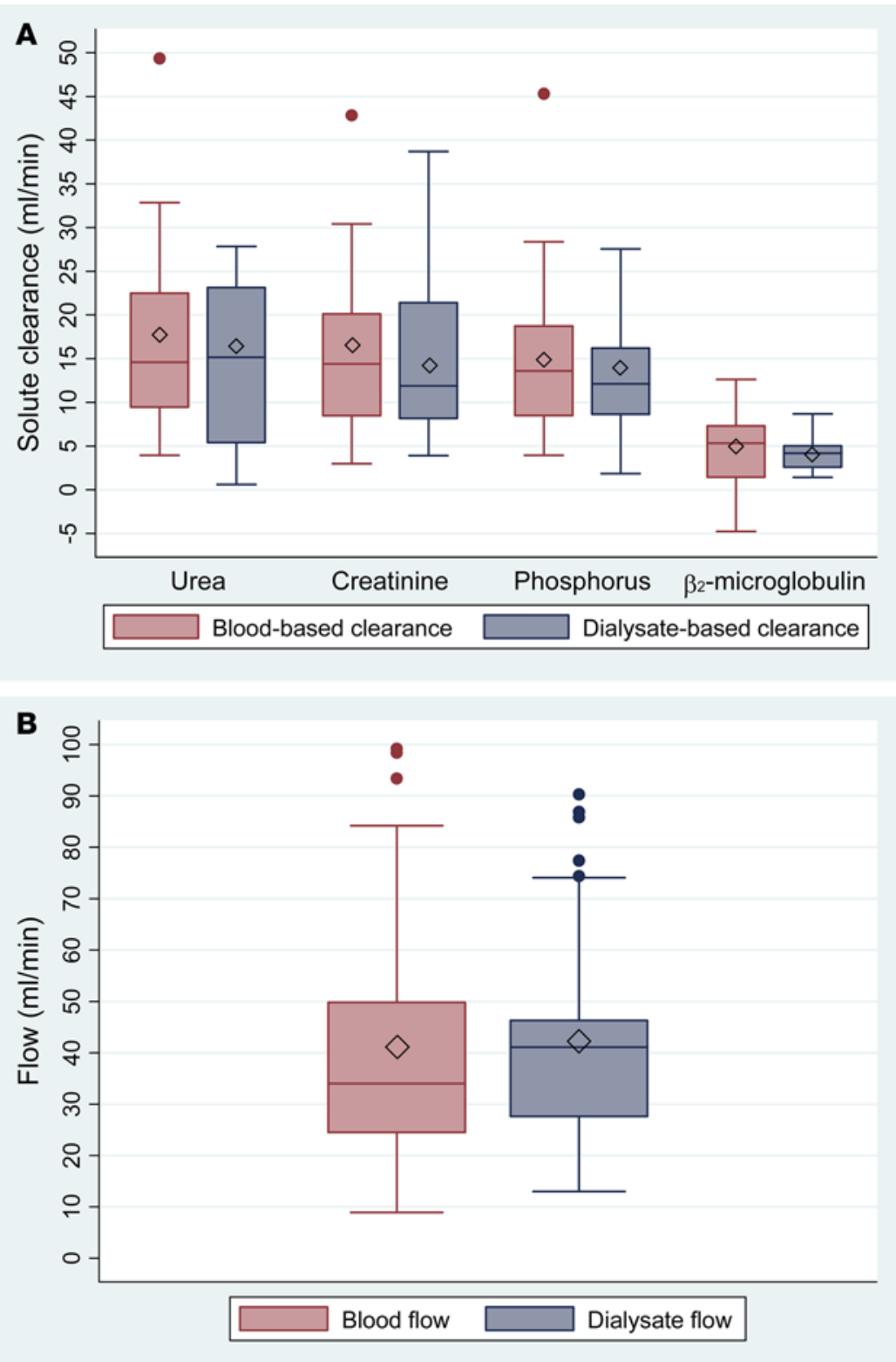

Figure 3. Summary of small solute and middle molecule clearance, blood flow, and dialysate flow. (A) Plasma urea, creatinine, phosphorus, and $\beta_{2}$-microglobulin clearance $(\mathrm{ml} / \mathrm{min})$. (B) Blood and dialysate flow $(\mathrm{ml} / \mathrm{min})$. Horizontal lines within boxes indicate median values, diamonds indicate mean values, top and bottom margins of boxes represent 25 th and 75 th percentile values, and whiskers indicate the range of values within 1.5 multiples of the interquartile range.

than utilized in conventional forms of hemodialysis. Given the capability of the WAK to operative continuously, less aggressive hourly clearances were targeted to minimize solute flux during the treatment period. We found that, as anticipated, solute clearance varied in parallel with blood flow, and clearances of urea, creatinine, and phosphorus were similar at each study time point. Overall, we observed that urea clearances were higher than those for creatinine, though this was true only for a subset of study participants. Higher dialytic clearance of urea compared with creatinine is expected due to the more rapid equilibration of urea between red blood cells and plasma, a principle that has been demonstrated in prior investigations (40-43). In contrast, though permeability of conventional dialyzer membranes is lower for inorganic phosphorus than for creatinine, prior studies have generally shown similar dialytic clearance of phosphorus compared with creatinine $(40,44,45)$. This may due to uneven phosphate distribution among different body compartments, with phosphate clearance during the dialytic procedure limited by the phosphorus transfer rate from the intracellular space to the plasma (46). The findings for comparative solute clearances observed in our study are similar to those from prior investigations, including similar time-varying clearances of phosphorus and creatinine for most patients. Although serum phosphorus remained normal in the absence of phosphate-binding medications or dietary restrictions, serum phosphorus rose for some patients over the course of the 24-hour study period. We also observed limited clearance of the middle-molecular weight solute $\beta_{2}$-microglobulin at the blood and dialysate flows achieved in this study. These data are, however, consistent with previously reported data using the WAK (23), suggesting that these lower clearances might be related to the lower blood flow used in this trial. We observed that overall mean blood flow diminished somewhat toward the end of the 24-hour treatment period, largely driven by a substantial drop in the blood flow for a single subject that was possibly related to either unintended variability in battery or shuttle pump function or to the effect of dialysate carbon dioxide gas accumulation on pump efficiency. Planned refinements of the WAK in the near future may increase solute clearances to approximate even greater kidney function by improving pump function through mechanical components redesign.

Patients enrolled in our study reported significantly greater treatment satisfaction during the WAK treatment period compared with ratings of care during periods of conventional in-center hemodialysis treatment. This finding is of particular importance, given a growing emphasis on enhancing patient-centeredness of care in all aspects of the US healthcare system. Providing patient-centered care - care that 


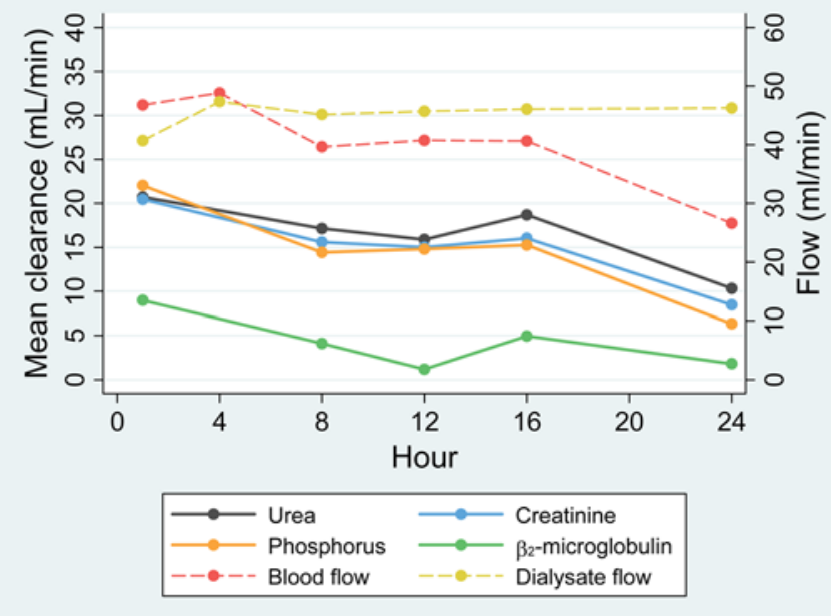

Figure 4. Mean small solute and middle molecule clearances, blood flow, and dialysate flow over the 24-hour wearable artificial kidney treatment period. Mean plasma creatinine, urea, phosphorus, and $\beta_{2}$-microglobulin clearance $(\mathrm{ml} / \mathrm{min})$ calculated using the blood-based Fick equation are shown as solid lines, with values corresponding to the left-sided $y$ axis. Blood and dialysate flow $(\mathrm{ml} / \mathrm{min})$ are shown as dashed lines, with values corresponding to the right-sided $y$ axis.

is in response to patients' preferences, values, and needs - is 1 of the 6 domains of quality of care advocated by the Institute of Medicine and is incentivized by the US Centers for Medicare and Medicaid services in designing quality incentive programs for the ESRD population $(47,48)$. Prior studies of extended-hours and/or frequent hemodialysis therapies have suggested potential improvements in health-related quality of life compared with conventional in-center hemodialysis therapy $(8,49-51)$. Additionally, previous mixed-methods assessments of patient and family preferences have shown that patients and caregivers highly value treatments that can be performed at home and that maximize independence and the ability to travel (52-54). Although the duration of exposure to the WAK was short and the study subjects represented a particularly motivated group of patients, our finding that treatment satisfaction scores for the WAK were higher in nearly every domain assessed is nonetheless an important preliminary result that deserves further investigation in future studies of the WAK.

There were no serious adverse events during the course of the study. We did not observe significant changes in serum haptoglobin, lactate dehydrogenase, or blood hematocrit, suggesting that no clinically significant hemolysis occurred over the course of the 24-hour WAK treatment. Similarly, we did not observe any clinically significant clotting of blood in the device in 6 of the 7 study subjects, even with lower hourly heparin infusions than commonly administered during conventional hemodialysis treatments. In the 1 subject in whom clotting occurred, this was likely attributable to a mechanical kinking of tubing that occurred during ambulation. Further studies are needed to clarify risk factors for clotting and optimal anticoagulation for patients treated with the WAK.

Our study had several limitations. The substantial production of carbon dioxide bubbles in the dialysate circuit was consistent with the observations of a previous study using the same device (21). Though this was anticipated, the venting mechanism used did not fully solve this problem, and manual removal of carbon dioxide gas from the dialysate circuit by study investigators using a lure-lock syringe was required for some patients. We observed intermittent variability in WAK blood and dialysate flow for the majority of patients. This variability had a number of possible sources. First, kinking of tubing was observed in both the blood and dialysate circuits at a variety of different device locations that included attachment of the blood tubing to the dialyzer, the attachments of the dialysate tubing into and out of the sorbent columns, and attachment of the dialysate tubing to the dialyzer. Second, excessive production of carbon dioxide bubbles resulted in increased resistance in the dialysate circuit, which may have impacted both blood and dialysate flow due to dual function of the shuttle pump to drive blood and dialysate flow simultaneously. Third, we observed variability in the performance of the main pulsatile pump with respect to flows generated, which may have been due to inconsistent power output or suboptimal positioning of the blood and/or dialysate tubing over the pump mechanism. Intermittent discordant variations in blood and dialysate flow rates in a number of subjects were also likely due to tubing kinks and dialysate-side carbon dioxide bubbles leading to differential resistances to flow on each side of the circuit. Further redesign of the WAK components is planned prior to future studies. The redesign will include more effective degassing in the dialysis circuit through the use of larger venting mechanisms, use of less flexible tubing, and use of a manifold to reduce potential for line kinking. Additionally, the sorbent system may be redesigned to include materials that may adsorb urea, avoid the need for urease activity, and thus substantially reduce carbon dioxide gas production in the dialysate circuit. Redesign of WAK components will be aimed at ensuring ease of use and reliability of the operation of the device, given that the ultimate goal is for treatment with the WAK to be self-administered independently by patients and caregivers in the home environment.

While undergoing treatment, subjects were observed in a highly monitored inpatient setting, which 

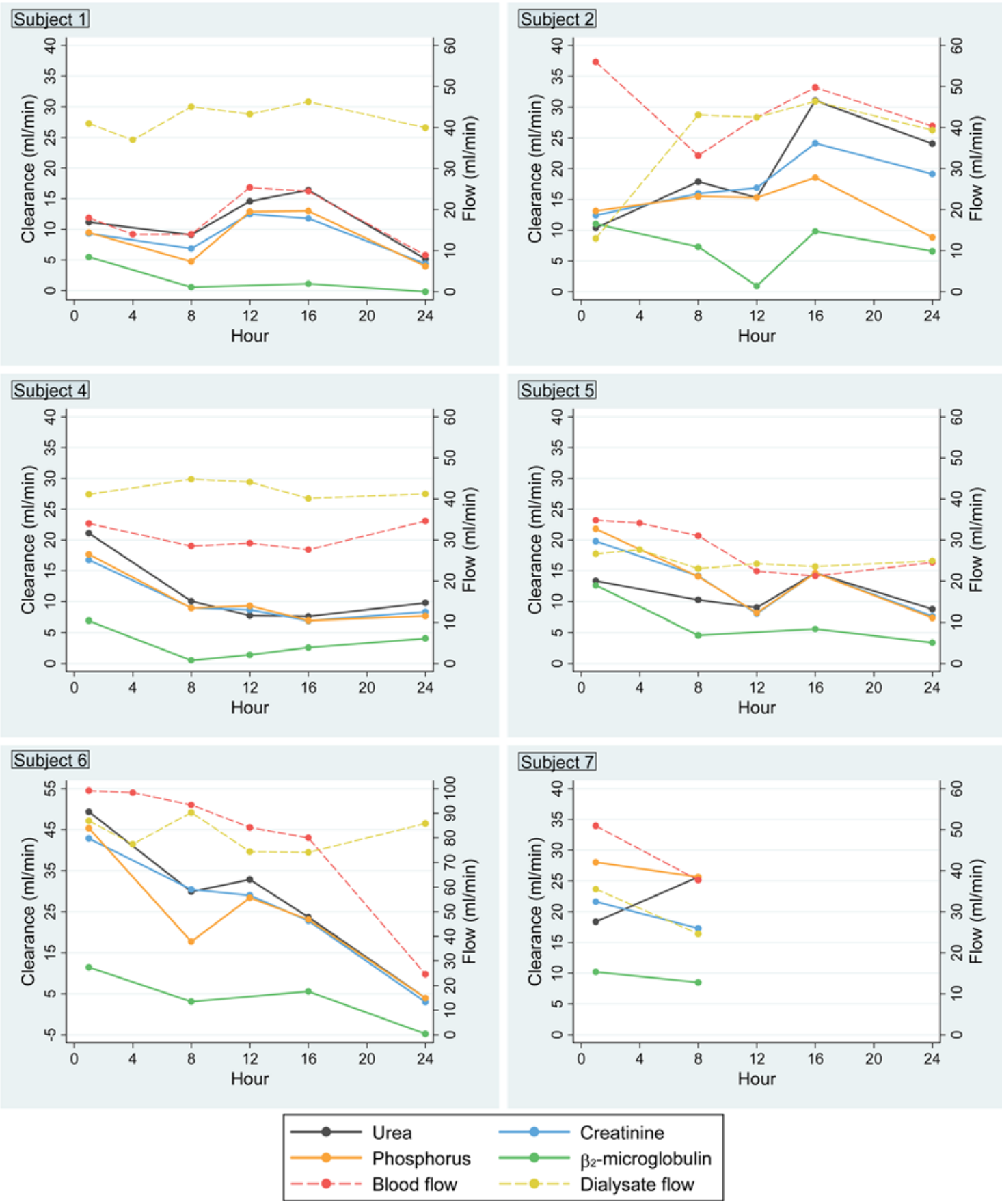

Figure 5. Time-varying small solute and middle molecule clearances, blood flow, and dialysate flow for individual subjects over the 24-hour wearable artificial kidney treatment period. Plasma creatinine, urea, phosphorus, and $\beta_{2}$-microglobulin clearance calculated using the blood-based Fick equation are shown as solid lines, with values corresponding to the left-sided $y$ axis. Blood and dialysate flow are shown as dashed lines, with values corresponding to the right-sided $y$ axis. Data are shown for the 6 individuals who completed $\geq 8$ hours of study treatment.

included hourly assessments of hemodynamic parameters and continuous cardiac telemetry. The long-term safety of continuous treatment with the WAK has not been established yet. Longer-term studies treating patients in the outpatient and home environment are necessary to address safety issues during ambulation and the home operation of the device by patients and to incorporate additional human factor elements.

In conclusion, the WAK is a dialysis technology with the potential to radically change the way dialysis therapy is delivered. As demonstrated in this FDA-approved exploratory clinical trial, 24-hour treatment of individuals with ESRD with a wearable, miniaturized, sorbent-based hemodialysis system is well-tolerated 


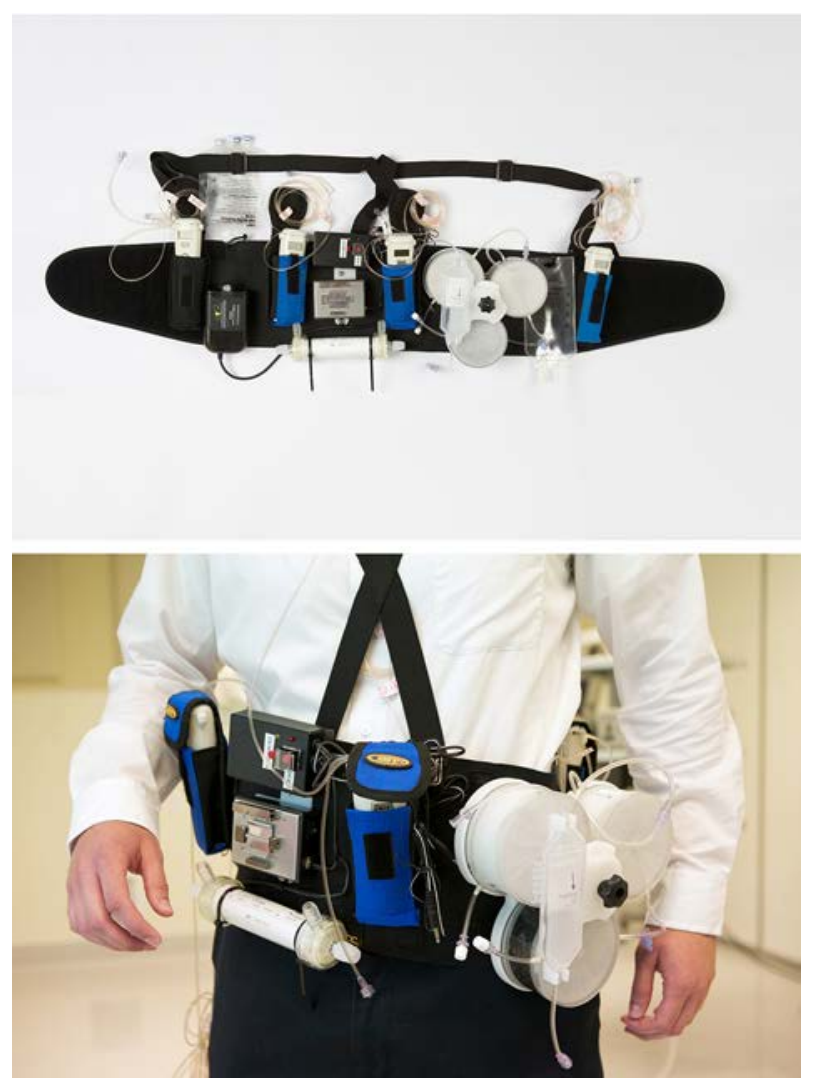

Figure 6. Prototype wearable artificial kidney. The prototype device assembled and worn and fastened in position.

and results in effective electrolyte homeostasis, solute clearance, and volume removal. Despite a variety of device-related technical complications encountered, this study serves as proof of concept of the WAK as an innovative alternative dialysis technology that has the potential to change the way ESRD is treated through enhancing patient choice and optimizing health-related quality of life for patients with ESRD.

\section{Methods}

Study subjects. Study subjects were recruited from outpatient dialysis facilities from November 2014 through April 2015. Subjects were at least 21 years of age, with a diagnosis of ESRD. They were undergoing maintenance hemodialysis thrice weekly for at least 3 months prior to enrollment. They weighed between 45 and 100 kilograms, with vascular access through a functioning double lumen tunneled dialysis catheter. Exclusion criteria included anticipated living related donor kidney transplant within 2 months, a history of recent cardiovascular events or hemodynamic instability, or current active infection. Other eligibility criteria are provided in the Supplemental Methods.

WAK treatment. For the WAK treatment period, all subjects were admitted to an inpatient hospital unit and were monitored continuously by study physicians and nurses. Prior to initiation of the 24-hour treatment with the WAK, each subject received a 4-hour conventional hemodialysis session according to the subject's routine clinical prescription.

Following a 2-hour treatment-free transition period, subjects were connected to the WAK (Blood Purification Technologies Inc.) (Figure 6) using an existing tunneled dialysis catheter. The WAK comprises a miniaturized wearable sorbent-based hemodialysis system utilizing a commercially available dialyzer (Gambro Polyflux $6 \mathrm{H}$, Baxter) with an effective surface area of $0.6 \mathrm{~m}^{2}$ and blood compartment volume of $52 \mathrm{ml}$. A pulsatile pump powered by a rechargeable battery with dual-channel separated circuits propels blood and dialysate in a counter-current fashion through the dialyzer $(20,55)$. Dialysate is regenerated using 3 sorbent-containing cartridges connected in series and containing, in order, urease (65 grams), zirconium phosphate (600 grams), hydrous zirconium oxide (51 grams), and activated charcoal (153 grams) (Figure 7). The sorbent system uses urease (derived from jack beans and bound to insoluble aluminum oxide) to convert urea into ammonia and carbonate, which, in the presence of hydrogen ions, forms carbon dioxide. The ammonia is subsequently adsorbed by the zirconium phosphate layer along with other cations, such as calcium, magnesium, and potassium, in exchange for sodium and hydrogen, which are released. The carbon dioxide is vented into the atmosphere as a gas through a semipermeable degassing bubble removal system (membrane surface area $17.7 \mathrm{~cm}^{2}, 3 \mathrm{M}$ ). The hydrated zirconium oxide layer removes heavy metals, such as copper and lead, if present and also adsorbs anions, such as phosphate, in exchange for acetate. Other medium-sized solutes and organic compounds are adsorbed by charcoal in the final sorbent column. The approximate total volume of the blood circuit is $65 \mathrm{ml}$ and of the dialysate circuit is $375 \mathrm{ml}$. Additional auxiliary micropumps (Sorenson Medical Products Inc.) control ultrafiltration, heparin infusion, and calcium acetate, magnesium acetate, and sodium bicarbonate infusion into the dialysate circuit. An ultrasonic bubble detector on the arterial blood tubing and an optical blood leak detector on the dialysate tubing (Introtek) trigger an audible alarm and a pause of the main pump. Wetness sensors (Economical Liquid Sensor, Cole-Palmer) applied to the arterial and venous catheter connection sites detect external blood leak. The total weight of the device, including the dialysate, is $5 \mathrm{~kg}$. "The WAK components used for testing in this trial were provided by Blood Purification Technologies Inc.

Vital signs, symptoms, and adverse events were recorded hourly, and electrocardiographic telemetry monitoring was carried out continuously. Subjects were encouraged to eat and drink a normal diet, without restric- 
Table 3. Subject ratings of treatment satisfaction comparing conventional hemodialysis with the WAK

\begin{tabular}{|c|c|c|c|c|}
\hline RTSQ item & Item content & $\begin{array}{l}\text { Conventional } \\
\text { hemodialysis }^{A}\end{array}$ & WAK $^{A}$ & P value ${ }^{\mathrm{B}}$ \\
\hline 1 & Satisfaction with treatment & 4.1 & 5.1 & 0.02 \\
\hline 3 & Satisfaction with treatment side effects & 3.4 & 5.7 & 0.003 \\
\hline 4 & Satisfaction with treatment-related demands & 3.9 & 4.7 & 0.22 \\
\hline 5 & Convenience of treatment & 4.0 & 4.9 & 0.04 \\
\hline 8 & Satisfaction with understanding of treatment & 5.1 & 5.7 & 0.23 \\
\hline 9 & Satisfaction with time taken by treatment & 2.7 & 5.0 & $<0.001$ \\
\hline 10 & Discomfort or pain involved with treatment & 3.9 & 5.0 & 0.04 \\
\hline 11 & How well treatment fits in with lifestyle & 3.1 & 5.7 & 0.002 \\
\hline 12 & Would you recommend this treatment to others & 3.9 & 5.9 & 0.06 \\
\hline
\end{tabular}

WAK, wearable artificial kidney; RTSQ, Renal Treatment Satisfaction Questionnaire. Andividual item scales are from 0 (lowest satisfaction) to 6 (highest satisfaction). ${ }^{\mathrm{B}} \mathrm{P}$ value from paired $t$ test. ${ }^{\mathrm{C}}$ Summary score calculated by summing individual item scores.

tion of fluid, sodium, potassium, or phosphorus content. At the beginning of WAK therapy, each subject was given an initial heparin bolus of 2,000 units and started on a continuous heparin infusion at 500 units per hour. The heparin infusion rate was adjusted or additional heparin boluses were given according to the clinical judgment of the study investigator at the bedside, including assessment of partial thromboplastin time (PTT) and the appearance of any clots in the blood tubing or dialyzer. The goal PTT was determined by the bedside study investigator and ranged from 50 to 80 seconds. The initial ultrafiltration rate was set at $50 \mathrm{ml}$ per hour and was adjusted hourly at the discretion of the treating physician based on observed vital sign parameters as well as observed dietary and fluid intake. All regularly prescribed home medications with the exception of phosphorus-binding agents were continued. Subjects were encouraged to ambulate with study staff assistance while receiving treatment with the device and also to sleep during night time hours.

Analytical procedures. Blood samples obtained at predetermined time points were analyzed at the bedside (i-STAT, Abbot) for safety purposes and in the hospital's clinical laboratory. Serum chemistries, bilirubin, lactate dehydrogenase, and haptoglobin were measured using a UniCel DxC 600 or 800 (Beckman Coulter), and complete blood count was measured using a Sysmex XE-5000 (Sysmex). Coagulation parameters were measured for the purpose of heparin titration using a Stago STA Compact analyzer (Diagnostica Stago Inc.). $\beta_{2}$-Microglobulin concentrations were measured on a Vmax Spectrophotometer (Molecular Devices) using a competitive binding enzyme immunoassay (R\&D Systems). To assess ammonia saturation of the sorbent cartridges, dialysate samples were tested hourly for ammonia accumulation (Sorb Technologies Inc.), with appearance of ammonia used as an indicator of sorbent column saturation. Detection of ammonia at a concentration of $5 \mathrm{mg} \%$ in any study subject prompted replacement of the 3-cartridge sorbent system.

Patient satisfaction with the WAK was assessed using the 13-item RTSQ (Health Psychology Research Limited) (56). The RTSQ has been developed and validated in patients with ESRD and has high internal reliability (Cronbach's $\alpha=0.93$ ). Patients completed ratings of conventional hemodialysis using the RTSQ during a screening visit prior to WAK treatment and completed ratings of the WAK at the first study visit following completion of the WAK protocol.

Statistics. Baseline demographic and laboratory characteristics were summarized. Changes in biochemical measurements from baseline were analyzed using analysis of variance for test of trend. Time-averaged concentrations (TACs) of BUN and $\beta_{2}$-microglobulin were calculated for each patient for a 48-hour period encompassing a conventional hemodialysis session as TAC $=\left[T_{d}\left(C_{1}+C_{2}\right)+I_{d}\left(C_{2}+C_{3}\right)\right] / 2\left(T_{d}+I_{d}\right)$, where $C_{1}$ is the venous concentration at the initiation of dialysis, $C_{2}$ is the concentration at the end of dialysis, $C_{3}$ is the concentration at the beginning of the next dialysis, $T_{d}$ is the session duration, and $I_{d}$ is the interdialytic interval (57). TACs for conventional hemodialysis were then compared with weighted average concentrations for the 24-hour period encompassing WAK treatment using the paired 2-tailed Student's $t$ test. 


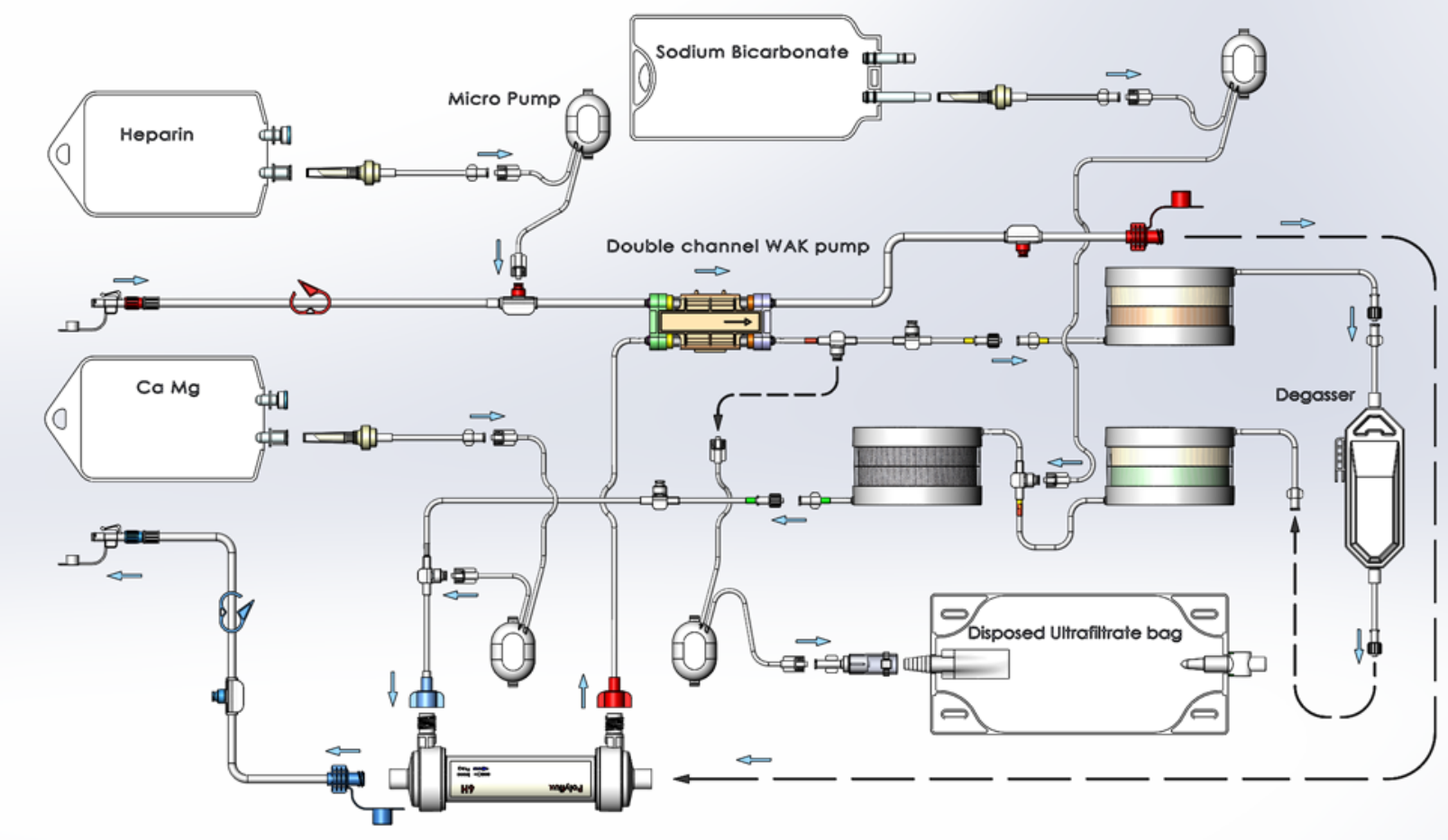

Figure 7. Detailed schematic flow diagram of WAK blood and dialysate circuits. $\mathrm{ZP}$, zirconium phosphate; $\mathrm{HZO}$, hydrous zirconium oxide; $\mathrm{CO}_{2} \mathrm{EF}$, semipermeable degassing bubble removal mechanism; WAK, wearable artificial kidney.

Clearances of urea, creatinine, inorganic phosphorus, and $\beta_{2}$-microglobulin during WAK treatment were calculated using two separate methods. Using blood side kinetics $(58,59)$, clearances were calculated according to the Fick principle as blood-based clearance $\left(K_{B}\right)=Q_{B} \times\left(C_{i n}-C_{o u t}\right) \times(1-H c t) / C_{i n}$, where $Q_{B}$ is the blood flow rate $(\mathrm{ml} / \mathrm{min}), C_{i n}$ is the blood concentration of solute entering the dialyzer, $C_{\text {out }}$ is the blood concentration of solute exiting the dialyzer, and Hct is the blood hematocrit. For 2 subjects, blood flows were not recorded at the 24-hour time point and were imputed as the 23 -hour values. Using dialysate-side kinetics $(58,60)$, solute clearance was also calculated from the ratio of mass removal rate to blood concentration as dialysate-based clearance $\left(K_{D}\right)=C_{\text {Dialysate }} \times Q_{D} / C_{\text {Blood }}$, where $Q_{D}$ is the dialysate flow rate $(\mathrm{ml} / \mathrm{min}) ; C_{\text {Dialssate }}$ is the dialysate solute concentration, calculated as the difference between the dialysate solute measurements before and after dialyzer; and $C_{\text {Blood }}$ is the blood concentration of solute entering the dialyzer. All data were included in the analyses, including data obtained during periods in which technical problems occurred with the WAK. Individual item treatment satisfaction scores and an overall summary score from the RTSQ for conventional hemodialysis and WAK treatment were compared using the paired 2-tailed Student's $t$ test. For all analyses, a $P$ value less than 0.05 was considered significant. All analyses were performed using Stata, version 11 (StataCorp LP).

Study approval. This study was performed in accordance with the Declaration of Helsinki and was approved by the University of Washington Institutional Review Board. All subjects provided written informed consent before study enrollment.

\section{Author contributions}

VG, LK, and JH conceived of the study, participated in its design and coordination, participated in interpretation of the data, and helped draft the manuscript. MBR participated in coordination and data collection for the study, performed the primary statistical analysis, interpreted the data, and drafted the manuscript. $\mathrm{SB}, \mathrm{RM}$, and NCS contributed to coordination of the study, design of the analytic plan, and interpretation 
of the data. LL, JK, MB, and CE participated in design of the study, study coordination and troubleshooting, and analysis and interpretation of the final data. All authors read, edited, provided critical review, and approved the final manuscript.

\section{Acknowledgments}

This study was supported by an unrestricted gift from the not-for-profit Wearable Artificial Kidney Foundation. Use of the WAK device for the purpose of this study was provided by the study sponsor, Blood Purification Technologies Inc. The study sponsor had no role in the analysis or interpretation of the data, in the writing of the manuscript, or in the decision to submit the manuscript for publication. M.B. Rivara is supported by a grant from the National Institute of Diabetes and Digestive and Kidney Diseases (T32DK007467). Credit for study photographs is to University of Washington School of Medicine/ Stephen Brashear. The authors would like to gratefully acknowledge the WAK dialysis nurse team for providing patient care: Yun Sue Choi, Chantelle Dykstra, Cara Havens, Irene Krall, Kristin McNeill, Laura Moffett, and Carol Moore. Additionally, the authors would like to acknowledge Lisa Anderson and Linda Manahan for study coordination, John Ruzinski and Denise Rock for laboratory management, and the study subjects and their families for their participation and enthusiasm. The RTSQ was used with permission of Clare Bradley and under license from Health Psychology Research Limited, Surrey, United Kingdom. Finally, the authors would like to thank the members of the data safety monitoring board for their time and efforts: Nathan W. Levin, Andre A. Kaplan, and Susanne May.

Address correspondence to: Jonathan Himmelfarb, 325 Ninth Avenue, Box 359606, Seattle, Washington 98104, USA. Phone: 206.616.8574; E-mail: jhimmelfarb@nephrology.washington.edu.

1. Thomas B, et al. Maintenance Dialysis throughout the World in Years 1990 and 2010. J Am Soc Nephrol. 2015;26(11):2621-2633.

2. Saran R, et al. US Renal Data System 2014 Annual Data Report: epidemiology of kidney disease in the United States. Am $J$ Kidney Dis. 2015;66(1 suppl 1):Svii, S1-Svii,305.

3. Longenecker JC, et al. Traditional cardiovascular disease risk factors in dialysis patients compared with the general population: the CHOICE Study. J Am Soc Nephrol. 2002;13(7):1918-1927.

4. Cheung AK, et al. Atherosclerotic cardiovascular disease risks in chronic hemodialysis patients. Kidney Int. 2000;58(1):353-362.

5. Chiu YW, Teitelbaum I, Misra M, de Leon EM, Adzize T, Mehrotra R. Pill burden, adherence, hyperphosphatemia, and quality of life in maintenance dialysis patients. Clin J Am Soc Nephrol. 2009;4(6):1089-1096.

6. Noori N, Kalantar-Zadeh K, Kovesdy CP, Bross R, Benner D, Kopple JD. Association of dietary phosphorus intake and phosphorus to protein ratio with mortality in hemodialysis patients. Clin J Am Soc Nephrol. 2010;5(4):683-692.

7. Noori N, et al. Dietary potassium intake and mortality in long-term hemodialysis patients. Am J Kidney Dis. 2010;56(2):338347.

8. FHN Trial Group, et al. In-center hemodialysis six times per week versus three times per week. $N$ Engl J Med. 2010;363(24):2287-2300.

9. Culleton BF, et al. Effect of frequent nocturnal hemodialysis vs conventional hemodialysis on left ventricular mass and quality of life: a randomized controlled trial. JAMA. 2007;298(11):1291-1299.

10. Chan CT, Floras JS, Miller JA, Richardson RM, Pierratos A. Regression of left ventricular hypertrophy after conversion to nocturnal hemodialysis. Kidney Int. 2002;61(6):2235-2239.

11. Johansen KL, et al. Survival and hospitalization among patients using nocturnal and short daily compared to conventional hemodialysis: a USRDS study. Kidney Int. 2009;76(9):984-990.

12. Lacson E, et al. Survival with three-times weekly in-center nocturnal versus conventional hemodialysis. J Am Soc Nephrol. 2012;23(4):687-695.

13. Nesrallah GE, et al. Intensive hemodialysis associates with improved survival compared with conventional hemodialysis. $J$ Am Soc Nephrol. 2012;23(4):696-705

14. Powell JR, et al. Ten years experience of in-center thrice weekly long overnight hemodialysis. Clin J Am Soc Nephrol. 2009;4(6):1097-1101.

15. Lindsay RM, Heidenheim PA, Nesrallah G, Garg AX, Suri R, Daily Hemodialysis Study Group London Health Sciences Centre. Minutes to recovery after a hemodialysis session: a simple health-related quality of life question that is reliable, valid, and sensitive to change. Clin J Am Soc Nephrol. 2006;1(5):952-959.

16. Weisbord SD, et al. Prevalence, severity, and importance of physical and emotional symptoms in chronic hemodialysis patients. J Am Soc Nephrol. 2005;16(8):2487-2494.

17. Johansen KL, Chertow GM, da Silva M, Carey S, Painter P. Determinants of physical performance in ambulatory patients on hemodialysis. Kidney Int. 2001;60(4):1586-1591.

18. Merkus MP, et al. Quality of life over time in dialysis: The Netherlands Cooperative Study on the Adequacy of Dialysis1. Kidney Int. 1999;56(2):720-728.

19. Bao Y, Dalrymple L, Chertow GM, Kaysen GA, Johansen KL. Frailty, dialysis initiation, and mortality in end-stage renal disease. Arch Intern Med. 2012;172(14):1071-1077.

20. Gura V, Macy AS, Beizai M, Ezon C, Golper TA. Technical breakthroughs in the wearable artificial kidney (WAK). Clin J Am 
Soc Nephrol. 2009;4(9):1441-1448.

21. Davenport A, Gura V, Ronco C, Beizai M, Ezon C, Rambod E. A wearable haemodialysis device for patients with end-stage renal failure: a pilot study. Lancet. 2007;370(9604):2005-2010

22. Gura V, et al. A wearable hemofilter for continuous ambulatory ultrafiltration. Kidney Int. 2007;73(4):497-502.

23. Gura V, Davenport A, Beizai M, Ezon C, Ronco C. Beta2-microglobulin and phosphate clearances using a wearable artificial kidney: a pilot study. Am J Kidney Dis. 2009;54(1):104-111.

24. Food and Drug Administration, Center for Devices and Radiological Health. Innovation Challenge: End-Stage Renal Disease. FDA Web site. http://www.fda.gov/AboutFDA/CentersOffices/OfficeofMedicalProductsandTobacco/CDRH/CDRHInnovation/InnovationPathway/ucm286140.htm. Updated July 30, 2015. Accessed May 12, 2016.

25. Jacobsen SC, Stephen RL, Bulloch EC, Luntz RD, Kolff WJ. A wearable artificial kidney: functional description of hardware and clinical results. Proc Clin Dial Transplant Forum. 1975;5:65-71.

26. Neff MS, Sadjadi S, Slifkin R. A wearable artificial glomerulus. Trans Am Soc Artif Intern Organs. 1979;25:71-73.

27. Blumenkrantz MJ, et al. Applications of the Redy sorbent system to hemodialysis and peritoneal dialysis. Artif Organs. 1979;3(3):230-236.

28. Murisasco A, et al. A continuous hemofiltration system using sorbents for hemofiltrate regeneration. Clin Nephrol. 1986;26(suppl 1):S53-S57.

29. Ronco C, Fecondini L. The Vicenza wearable artificial kidney for peritoneal dialysis (ViWAK PD). Blood Purif. 2007;25(4):383-388.

30. Lee DB, Roberts M. A peritoneal-based automated wearable artificial kidney. Clin Exp Nephrol. 2008;12(3):171-180.

31. Kim S, Fissell WH, Humes DH, Roy S. Current strategies and challenges in engineering a bioartificial kidney. Front Biosci (Elite Ed). 2015;7:215-228.

32. Tentori $\mathrm{F}$, et al. Longer dialysis session length is associated with better intermediate outcomes and survival among patients on in-center three times per week hemodialysis: results from the Dialysis Outcomes and Practice Patterns Study (DOPPS). Nephrol Dial Transplant. 2012;27(11):4180-4188.

33. Mucsi I, Hercz G, Uldall R, Ouwendyk M, Francoeur R, Pierratos A. Control of serum phosphate without any phosphate binders in patients treated with nocturnal hemodialysis. Kidney Int. 1998;53(5):1399-1404.

34. Walsh M, Culleton B, Tonelli M, Manns B. A systematic review of the effect of nocturnal hemodialysis on blood pressure, left ventricular hypertrophy, anemia, mineral metabolism, and health-related quality of life. Kidney Int. 2005;67(4):1500-1508

35. Saran R, et al. Longer treatment time and slower ultrafiltration in hemodialysis: associations with reduced mortality in the DOPPS. Kidney Int. 2006;69(7):1222-1228.

36. Flythe JE, Kimmel SE, Brunelli SM. Rapid fluid removal during dialysis is associated with cardiovascular morbidity and mortality. Kidney Int. 2011;79(2):250-257.

37. Jefferies HJ, Virk B, Schiller B, Moran J, McIntyre CW. Frequent hemodialysis schedules are associated with reduced levels of dialysis-induced cardiac injury (myocardial stunning). Clin J Am Soc Nephrol. 2011;6(6):1326-1332.

38. Cornelis $\mathrm{T}$, et al. Acute hemodynamic response and uremic toxin removal in conventional and extended hemodialysis and hemodiafiltration: a randomized crossover study. Am J Kidney Dis. 2014;64(2):247-256.

39. Greene T, et al. Solute clearances and fluid removal in the frequent hemodialysis network trials. Am J Kidney Dis. 2009;53(5):835-844.

40. Debowska M, Wojcik-Zaluska A, Ksiazek A, Zaluska W, Waniewski J. Phosphate, urea and creatinine clearances: haemodialysis adequacy assessed by weekly monitoring. Nephrol Dial Transplant. 2015;30(1):129-136.

41. Clark WR, Leypoldt JK, Henderson LW, Mueller BA, Scott MK, Vonesh EF. Quantifying the effect of changes in the hemodialysis prescription on effective solute removal with a mathematical model. J Am Soc Nephrol. 1999;10(3):601-609.

42. Schneditz D, Platzer D, Daugirdas JT. A diffusion-adjusted regional blood flow model to predict solute kinetics during haemodialysis. Nephrol Dial Transplant. 2009;24(7):2218-2224.

43. Descombes E, Perriard F, Fellay G. Diffusion kinetics of urea, creatinine and uric acid in blood during hemodialysis. Clinical implications. Clin Nephrol. 1993;40(5):286-295.

44. Sedlacek M, Dimaano F, Uribarri J. Relationship between phosphorus and creatinine clearance in peritoneal dialysis: clinical implications. Am J Kidney Dis. 2000;36(5):1020-1024.

45. Basile C, et al. Removal of uraemic retention solutes in standard bicarbonate haemodialysis and long-hour slow-flow bicarbonate haemodialysis. Nephrol Dial Transplant. 2011;26(4):1296-1303.

46. Pohlmeier R, Vienken J. Phosphate removal and hemodialysis conditions. Kidney Int Suppl. 2001;78:S190-S194.

47. IOM (Institute of Medicine). Initial National Priorities for Comparative Effectiveness Research. Washington, DC, USA: The National Academies Press; 2009:

48. Office of the Federal Register, National Archives Records Administration. Medicare Program; End-Stage Renal Disease Prospective Payment System, Quality Incentive Program, and Durable Medical Equipment, Prosthetics, Orthotics, and Supplies. Fed Register. 2013;78(231):72155-72253.

49. Finkelstein FO, et al. At-home short daily hemodialysis improves the long-term health-related quality of life. Kidney Int. 2012;82(5):561-569.

50. Unruh ML, et al. Effects of 6-times-weekly versus 3-times-weekly hemodialysis on depressive symptoms and self-reported mental health: Frequent Hemodialysis Network (FHN) Trials. Am J Kidney Dis. 2013;61(5):748-758.

51. Hall YN, et al. Effects of six versus three times per week hemodialysis on physical performance, health, and functioning: Frequent Hemodialysis Network (FHN) randomized trials. Clin J Am Soc Nephrol. 2012;7(5):782-794.

52. Morton RL, et al. Dialysis modality preference of patients with CKD and family caregivers: a discrete-choice study. Am J Kidney Dis. 2012;60(1):102-111.

53. Morton RL, Tong A, Webster AC, Snelling P, Howard K. Characteristics of dialysis important to patients and family caregivers: a mixed methods approach. Nephrol Dial Transplant. 2011;26(12):4038-4046

54. Morton RL, Devitt J, Howard K, Anderson K, Snelling P, Cass A. Patient views about treatment of stage 5 CKD: a qualitative analysis of semistructured interviews. Am J Kidney Dis. 2010;55(3):431-440. 
55. Rambod E, Beizai M, Rosenfeld M. An experimental and numerical study of the flow and mass transfer in a model of the wearable artificial kidney dialyzer. Biomed Eng Online. 2010;9:21.

56. Barendse SM, Speight J, Bradley C. The Renal Treatment Satisfaction Questionnaire (RTSQ): a measure of satisfaction with treatment for chronic kidney failure. Am J Kidney Dis. 2005;45(3):572-579.

57. Hakim RM. Assessing the adequacy of dialysis. Kidney Int. 1990;37(2):822-832.

58. Wolf AV, Remp DG, Kiley JE, Currie GD. Artificial kidney function; kinetics of hemodialysis. J Clin Invest. 1951;30(10):1062-1070.

59. DeSoi CA, Umans JG. Phosphate kinetics during high-flux hemodialysis. J Am Soc Nephrol. 1993;4(5):1214-1218.

60. Claure-Del Granado R, et al. Toward the optimal dose metric in continuous renal replacement therapy. Int J Artif Organs. $2012 ; 35(6): 413-424$ 